\title{
Diagnostic applications of cell-free and circulating tumor cell-associated miRNAs in cancer patients
}

Bianca Mostert ${ }^{1}$, Anieta M. Sieuwerts ${ }^{2}$, John W.M. Martens ${ }^{2}$, Stefan Sleijfer ${ }^{1}$

Department of Medical Oncology, Erasmus Medical Center, Daniel den Hoed Cancer Center, Rotterdam,

Netherlands

${ }^{1}$ Daniel den Hoed Cancer Center, Laboratory of Clinical Tumor Immunology

${ }^{2}$ Josephine Nefkens Institute and Cancer Genomics Centre

\section{Bianca Mostert}

Erasmus MC, Daniel den Hoed Cancer Center, Room D2-62

Groene Hilledijk 301

3075 EA Rotterdam

Netherlands

Tel.: +31 107041324

Fax: +31107041005

Email: b.mostert@erasmusmc.nl

Corresponding author:

Anieta M. Sieuwerts

Erasmus MC, Josephine Nefkens Institute, Room BE-400

Dr. Molewaterplein 50

3015 GE Rotterdam

Netherlands

Tel.: +31107044372

Fax: +31107044377

Email: a.sieuwerts@erasmusmc.nl

John W.M. Martens

Erasmus MC, Josephine Nefkens Institute, Room BE-400

Dr. Molewaterplein 50

3015 GE Rotterdam

Netherlands

Tel.: +31 107044372

Fax: +31107044377

Email: j.martens@erasmusmc.nl

Stefan Sleijfer

Erasmus MC, Daniel den Hoed Cancer Center, Room G4-83

Groene Hilledijk 301,

3075 EA Rotterdam

Netherlands

Tel: +31 107041331

Fax: +31107041003

Email: s.sleijfer@erasmusmc.nl 


\section{Summary}

Circulating tumor cells (CTCs) have rapidly developed as important cancer biomarkers after their enumeration proved to be prognostic in metastatic breast, colorectal and prostate cancer, and their rise or decline after the first cycle of therapy showed to predict therapy response. Besides mere counting, CTCs can be isolated and subsequently analyzed using various molecular applications, including miRNA expression analysis. Recently, miRNA expression profiling in primary tumors has yielded promising results. However, establishing miRNA expression in the circulation likely has advantages over determination in primary tumor tissue, further augmenting the potential applications of miRNA determination in oncology. Additionally to CTC-associated miRNAs, free circulating miRNAs have been identified in whole blood, plasma and serum.

Since determination of miRNAs in peripheral blood, either cell-free or CTC-associated, is expected to become important in oncology, especially when linked to and interpreted together with epithelial CTCs, this review focuses on measuring miRNAs in the circulation of cancer patients.

\section{Keywords:}

Circulating tumor cells, miRNA, microRNA, diagnosis, prognosis, response prediction, drug target. 


\section{Introduction}

The implementation of assays enabling the detection of circulating tumor cells (CTCs) has sparked an additional boost of interest in blood-derived biomarkers for cancer patients. Numerous assays for CTC enumeration have been described lately and for one, the CellSearch ${ }^{\mathrm{TM}}$ Epithelial Cell test (Veridex LCC, Raritan, NJ), FDA-approval has been acquired for use as a prognostic factor when measured in patients with metastatic breast[1,2], colorectal[3] and prostate cancer[4]. Additionally to its application as a prognosticator prior to treatment start, enumeration of CTCs may also guide treatment decisions, as a rise or decline in the number of CTCs after the first cycle of chemotherapy predicts for therapy response earlier than conventional radiographic evaluation does[5].

Probably even more interesting than mere counting, CTCs can be isolated from the blood of cancer patients for further analysis. In the case of metastatic disease, analysis of metastatic tissue can be very informative. Often, a considerable amount of time has passed since the occurrence and resection of the primary tumor and in many cases systemic adjuvant treatment has been administered. This means that clinically relevant changes can have occurred in the geno- and phenotype of residual cancer cells, and these changes could and probably should affect treatment decisions. Therefore, characterization of metastatic tissue, rather than that of the primary lesion, may show a better association with outcome in cancer patients.

Clinicians are however understandably reluctant to perform invasive and complicated procedures to obtain tissue from patients for whom quality of life is a major concern. The isolation and subsequent characterization of CTCs provide the opportunity to bypass the problems associated with obtaining metastatic tissue, and serve as a 'liquid biopsy'. CTCs have already been characterized for presence of gene amplification[6-8] and genetic aberrations[9,10], for expression of proteins[11] and several mRNAs[12-14], and recently, also for expression of certain miRNAs(submitted manuscript).

In recent years, miRNAs have been revealed as key regulators of gene expression. Given this crucial role, it is not surprising that miRNA expression in primary tumor tissue associates with outcome in several studies. However, determination of miRNA expression in the peripheral circulation, either CTC-associated or as cell-free circulating molecules, likely has several advantages over determination in primary tumor tissue, thereby further augmenting the potential applications of miRNA determination in oncology.

In this review, we will discuss the measurement of cell-free and CTC-associated miRNAs present in the peripheral circulation, and give examples of the clinical applications of this upcoming research field. 


\section{MiRNAs}

MiRNAs (miRs) are small single-stranded RNA molecules, measuring 21-23 nucleotides in length, which have, since their discovery in 1993[15], been shown to play important roles in regulating gene expression[16]. Until recently, miRNAs were disregarded as degraded RNA fragments or non-translated small RNAs, but the discovery of their aberrant expression in a wide array of pathological events and their involvement in carcinogenesis has made them a hot topic in cancer research[17,18]. One of the big advantages of miRNAs is their stability, and it has been shown that cell-free miRNAs in body fluids are stable under harsh conditions such as high temperatures, extreme $\mathrm{pH}$ values, repeated freeze-thaw cycles and long-term storage[1924].They are well preserved, not only in blood, but also in tissues that have been formalin-fixed and paraffin-

embedded years before[25]. This enables the retrospective analysis of large tissue collections, providing researchers with massive amounts of information. The identification of miRNAs has yielded an exciting new array of easy accessible molecular features, which may be employed in diagnostic and therapeutic decision making in cancer patients.

\subsection{MiRNA biology}

In the nucleus, miRNAs are transcribed by RNA polymerase II into large polyadenylated, capped primary miRNA transcripts (pri-miRNAs)[18,26] (Figure 1). These pri-miRNAs are subsequently cleaved by a complex formed by the RNAse II enzyme Drosha and its binding partner DGCR8 (DiGeorge syndrome critical region 8, or Pasha) into precursor miRNAs (pre-miRNAs). These pre-miRNAs are 70-90 nucleotides in length and have an imperfect stem loop hairpin structure. They are transported into the cytoplasm by Exportin 5 where the hairpin precursors are cleaved by a complex formed by the RNAse III enzyme Dicer and its binding partner TRBP (HIV-1 transactivating response RNA binding protein); resulting in a small dsRNA duplex that contains both the mature miRNA strand and its complementary strand. The mature miRNA strand is then incorporated into a RNA-induced silencing complex (RISC), which inhibits the function of its target mRNA by mRNA degradation or, most commonly, by translational repression after binding of the RISC to the target mRNA. Additionally, miRNAs can increase the expression of their target mRNA[18,27].

\subsection{MiRNA function}


MiRNAs are estimated to regulate up to $30 \%$ of all protein-coding genes[28]. They regulate post-transcriptional gene expression in a sequence-specific manner, recognizing their mRNA target with the 5'-end of the mature miRNA strand, which is often referred to as the 'seed-sequence'[29]. After recognition of the target mRNA, regulation of gene expression can occur through two different mechanisms, depending upon the complementarity of the miRNA sequence with its target mRNA. When perfect base-paring homology exists between the miRNA and the mRNA, the RNA-mediated interference pathway is induced, which leads to cleavage of the mRNA by Argonaute, present in the RISC complex. When imperfect binding to partially complementary sequences in the 3'-untranslated region of target mRNAs occurs, which is more frequent than perfect binding, the target mRNA is regulated by repression of protein translation. Consequently, proteins are regulated by miRNAs without significantly affecting the corresponding mRNA expression levels. Such knowledge underscores the need to combine mRNA and miRNA data to generate improved predictive and prognostic models.

\section{MiRNAs in primary cancer and their potential applications}

MiRNAs are thought to play two distinctly different roles in carcinogenesis, functioning both as 'oncomirs' and as tumor suppressors. This hypothesis is supported by the observation that miRNA expression in tumors can

be up- or downregulated compared to normal tissue[30]. The miRNA expression profiling of tumors has provided many new insights into states of differentiation and lineages within different tumor types.

As a consequence of the crucial role of miRNAs in tumor biology, there is a broad range of potential applications of miRNA measurement in oncology. Besides being informative of tumor biology, miRNA signatures can also be a diagnostic tool, serve as prognostic factors, predictive factors, potential drug targets and as pharmacodynamic markers. All of these applications are possible in primary tumors and metastases, but the stability of miRNAs also enables their detection in the circulation. In this field, circulating miRNAs can serve as biomarkers that can be measured repeatedly and non-invasively in a wide array of cancer types.

Research to date has however mainly focused on primary tumor tissue. We will, without attempting to give a complete overview, provide examples of miRNAs being used as any of the aforementioned biomarkers.

\subsection{MiRNAs to identify cancer tissue origin}


MiRNAs can serve to determine the tissue of origin for cancers of unknown primary origin, as has been shown with a classifier based on 48 miRNAs[31,32]. This microarray-based classifier was generated on 205 primary tumors and 131 metastases of 22 different tumor origins. The classifier was validated in an independent test set, in which it reached an overall sensitivity of $72 \%$ and a specificity of $99 \%$. This application could be very informative in the still existing problem of metastatic cancer patients in whom no primary tumor can be identified, and for whom no standard chemotherapy exists.

\subsection{MiRNA expression profiles to classify cancers}

Lu and co-workers showed that miRNA profiles are surprisingly informative, reflecting the developmental lineage and differentiation state of the tumors. They not only observed a general down regulation of miRNAs in tumors compared to normal tissues, but were also able to successfully classify poorly differentiated tumors using miRNA expression profiles. Contrarily, messenger RNA profiles were highly inaccurate in classifying tumors when applied to the same samples[30]. Breast cancer is a notoriously heterogeneous disease, but miRNAs can help to identify the subtype origin of tumor cells, as was demonstrated by Sempere and coworkers using an in situ hybridization method to reveal the spatial distribution of miRNA expression in archived formalin-fixed, paraffin-embedded breast tumors[33].

\subsection{MiRNAs as prognostic factors}

Many investigators have focused on identifying miRNAs that can separate patient groups according to prognosis. It would be beyond the scope of this review to discuss all studies that have identified such prognostic miRNAs, and we refer to Ferracin et al.[34] for a complete and comprehensive overview.

\subsection{Predictive miRNAs}

Not many data have been generated identifying specific miRNAs that can predict response to systemic therapy. This is not surprising, as determining true predictive value of a miRNA requires studies designed very carefully specifically for that research question.

\subsubsection{Ovarian cancer}

In ovarian cancer, miR-214 has been identified as a miRNA involved in resistance to cisplatin, through targeting of PTEN[35]. In this study, 4 of the most differentially expressed miRNAs among a total of 515 miRNAs tested 
in 10 ovarian tumors and 10 normal cell line pools were further validated. MiR-214 was one of the most frequently upregulated miRNAs in 30 primary ovarian tumors; and its role in cisplatin resistance was elucidated by knocking down miR-214, causing increased PTEN protein expression and decreased Akt phosphorylation. Furthermore, the expression of miR-214 in miR-214-negative cell lines led to resistance to cisplatin-induced cell death, and subsequent knockdown of miR-214 resulted in increased sensitivity to cisplatin-induced cell death[35]. These promising results should be validated in patients treated with cisplatin before miR-214 can be used as a valid biomarker to predict cisplatin response.

\subsubsection{Non-small cell lung cancer}

This validation in patient samples was performed in a study looking at the predictive value of miR-128b expression on response to gefitinib, an EGFR inhibitor, in non-small cell lung cancer (NSCLC)[36]. MiR-128b was chosen based on its regulatory role for EGFR and the fact that loss of chromosome $3 p$, where miR-128b is located, is one of the most frequent and earliest events in lung carcinogenesis. An inverse relationship between miR-128b and EGFR expression was observed in NSCLC cell lines, and while EGFR expression as assessed by immunohistochemistry did not correlate with gefitinib response in 58 NSCLC patients, EGFR mutations and loss of miR-128b were associated with improved response to gefitinib. In multivariate analysis, only histology, line of treatment and loss of miR-128b, and not EGFR expression or mutation, were found to be predictive of response[36].

\subsubsection{Hepatocellular carcinoma (HCC)}

$\mathrm{Ji}$ et al. undertook a carefully designed study in three independent cohorts of a total of $455 \mathrm{HCC}$ patients, and identified miR-26 to be lower expressed in tumors than in paired noncancerous tissue[37]. Additionally, of the patients who were not treated with interferon, the control arm of the cohorts, those with lower expression of miR-26 in their tumor had a shorter overall survival. Contrarily, of the patients in the treatment arm of the cohorts who did receive interferon, those with lower miR-26 expression had an improved survival compared to patients with higher miR-26 expression. In multivariate analysis too, a significant interaction was observed between miR-26 expression and response to interferon therapy[37].

\subsubsection{Breast cancer}

While data have been generated on breast cancer cell lines[38,39] we recently selected 5 candidate predictive miRNAs from 249 miRNAs measured in a small discovery set of breast cancer specimens and analyzed their expression in an independent series of 246 ER-positive primary breast tumors. In multivariate analysis, higher 
expression of miR-30c was associated with benefit from first line tamoxifen monotherapy and longer progression-free survival[40].

\subsection{MiRNAs as drug targets}

Because of their pivotal role in cancer development, progression and treatment, several preclinical findings point at the great potential to use miRNA as drug targets, either by inhibiting overexpressed 'oncomirs' or replacing underexpressed tumor suppressor miRNAs. Inhibition of miR-21 has been shown to reduce tumor development and metastatic potential in breast cancer cells[41]. Inhibition of miR-21, combined with miR-200b, also enhanced response to gemcitabine in cholangiocarcinoma cells[42]. In breast cancer cells, reintroducing miR-205 resulted in improved response to tyrosine kinase inhibitors through HER3 silencing[43].

Finally, researchers have demonstrated that hepatocellular carcinoma cells have reduced expression of miR26 , while this miRNA is highly expressed in normal tissues. Re-expression of miR-26 caused cells to arrest in G1, probably through repression of cyclin D2 and cyclin E2. When administering miR-26 to a mice model using a adeno-associated viral vector, cancer cell proliferation was reduced and apoptosis increased[44].

\section{Measuring miRNAs in the circulation}

As depicted above, promising results have been obtained in primary tumor material with respect to miRNAs as cancer biomarkers. There are however a number of situations in which it is likely that the value of miRNAs can be further augmented by measuring miRNAs in blood, either as cell-free circulating miRNAs or as CTCassociated miRNAs. Below we will discuss the potential applications of circulating miRNAs.

\subsection{Circulating miRNAs can help to more accurately predict patient outcome}

Many studies have focused on identifying prognostic miRNAs in primary tumors, and these prognostic factors are now known for a large variety of tumor types. While these prognostic miRNAs do distinct those patients with a favorable outcome from those with an unfavorable outcome, measuring miRNAs in the primary tumor does not take into account two important things; not all cells in the primary tumor have the ability to metastasize, and the subset of spreading tumor cells might differ in genetic make-up. Secondly, at the time of metastatic disease, genetic characteristics of the remaining or relapsing tumor cells can differ from those of the primary tumor, because by the time a patient presents with metastatic disease, years might have gone by since first presentation, and various different anti-tumor treatments can have been administered. Both these factors can 
cause profound differences in genetic and epigenetic make-up between primary tumor and metastatic tissue. At the time of disseminated disease, optimally, metastatic tissue would be used to determine prognosis; however, acquiring such tissue can often only be done through painful and invasive procedures. Circulating tumor cells can serve as a 'liquid biopsy' representing the patient's tumor load, and thereby provide a unique opportunity to assess prognosis in real time.

Furthermore, the presence of miRNAs that are associated with the process of metastasis or epithelial-tomesenchymal transition, a process that is thought to be necessary for haematogenous spread of disease to occur, might identify those patients that already have distant micrometastases too small to diagnose otherwise.

\subsection{Circulating miRNAs to predict response to anti-tumor therapy}

When systemic therapy is warranted, either in the adjuvant or metastatic setting, the choice of first line treatment can be crucial for ultimate patient outcome. Depending on tumor type, various patient and tumor characteristics are taken into consideration when deciding on the optimal treatment, but still, for a proportion of patients, ineffective therapy is started. Especially in patients receiving targeted therapy, such as EGFR inhibitors, factors determining their benefit are still being discovered. While most attention has been given to predictive factors such as KRAS mutations and EGFR expression, it may be expected that miRNAs will turn out to play an equally important role, given their pivotal role in cancer progression. As discussed before, circulating miRNAs can be measured repeatedly, which is especially important for their use as a predictive factor. One can imagine wanting to administer a certain systemic therapy as second-line treatment, for which a predictive miRNA has been established. While this miRNA can be measured in the primary tumor, earlier administered systemic therapy can have affected the expression of this miRNA in residual cancer cells. It is, therefore, very conceivable that a treatment adapted to circulating tumor characteristics is more beneficial than a treatment based on primary tumor characteristics.

Whenever a certain treatment has been started based on miRNA expression data, re-assessment can occur each time the patient becomes refractory to the installed treatment. Acquired resistance to systemic anti-tumor treatment is a major problem in cancer treatment, and overcoming that resistance by administering targeted therapy based on changed tumor characteristics might greatly improve patients' prognosis.

\subsection{Circulating miRNAs as a monitorable drug target}


One of the big theoretical advances of targeting miRNAs is the ability to monitor their expression in the circulation. When anti-miRNA treatment is started, miRNA expression levels could be monitored in blood at various time points, and their increase could predict treatment resistance and warrant a switch in therapeutic regimen. Already, it was shown that the administration of intravenous anti-miR-16, anti-miR-122, anti-miR-192 and anti-miR-194 caused a decrease in the levels of the corresponding miRNAs across all organs in mice[45]. In this sense, circulating miRNAs could serve as combined drug targets and pharmacodynamic markers.

\section{Cell-free circulating miRNAs}

In view of the potential advantages of determining miRNA expression in the peripheral circulation over that in primary tumor tissue, several studies have already identified free circulating miRNAs that are expressed in the circulation of cancer patients. Importantly, most of these miRNAs were found to be differentially expressed between cancer patients and healthy donors. These miRNAs were found to be either diagnostic or prognostic, but little study has been done on their potential roles as predictive factors or drug targets. The main findings of the studies on circulating miRNAs in relation to diagnosis and prognosis are reviewed below and summarized according to 12 different primary tumor types in Table 1. We have focused on solid tumors for this review, and refer to Fabbri et al.[46] for a comprehensive review of the many research advances in the field of miRNAs in hematological malignancies.

\subsection{Carcinomas of unknown primary}

MiRNAs can serve to determine the tissue of origin for cancers of unknown primary origin, as has been shown with a classifier based on 48 miRNAs determined in primary or metastatic tumor tissue [31,32]. Lodes et al. focused on the evaluation of miRNA expression patterns in human serum for five types of human cancer, prostate, colon, ovarian, breast and lung, using a pan-human microRNA, high density microarray, and identified a serum classifier based on 28 circulating miRNAs able to separate cancer cases from normal individuals[47]. In serum of cancer patients, specific miRNA expression patterns for lung cancer and colorectal cancer have been identified[19], providing evidence that miRNAs present in the circulation contain fingerprints for various diseases.

\subsection{Breast cancer}


In 148 breast cancer patients and 44 healthy controls, seven candidate miRNAs were measured in whole blood by RT-PCR without a preceding enrichment step. All miRNAs could be measured in patients and controls alike, but miR-195 and let-7a were expressed higher in breast cancer patients than in controls, with a mean fold change of 19 and 11, respectively. Additionally, the levels of these two miRNAs decreased significantly after curative tumor resection[48].

The first study that reported circulating miRNAs as potential biomarkers of early stage breast cancer with different results for Caucasian American (CA) versus African American (AA) women, concerns the study of Zhao and co-workers. After comparing levels of circulating miRNAs in plasma samples of 20 patients with early stage breast cancer and 20 matched controls, they reported 17 upregulated and 14 downregulated miRNAs in the $10 \mathrm{CA}$ women and 9 upregulated and 9 downregulated miRNAs in the 10 AA women. Furthermore, they were able to link these differentially expressed miRNAs to specific pathways using target prediction algorithms [49].

In a larger study evaluating miR-21 expression in the serum of 102 breast cancer patients and 20 healthy controls, this miRNA was found to be higher expressed in patients, especially in stage IV breast cancer[23]. Recently, 4 breast cancer associated miRNAs were measured in the serum of 59 localized breast cancer patients after primary tumor surgery, 30 metastasized breast cancer patients and 29 healthy controls. MiR-10b, miR-34a and miR-155 discriminated metastasized breast cancer patients from controls, and the latter was higher expressed in localized breast cancer patients than healthy controls but also than metastasized breast cancer patients[50]. Another study measured three miRNAs (miR-16, miR-145 and miR-155) in the serum of 13 breast cancer patients and 8 healthy controls, but did not find a difference in expression between these two groups[51].

\subsection{Non-small cell lung cancer}

Hu et al. used serum of NSCLC patients to look for miRNAs that were differentially expressed between 30 patients with longer survival and 30 patients with shorter survival, matched by age, sex and stage. Eleven miRNAs were found to differ more than five-fold between the two groups, and four of those were confirmed by RT-PCR to be associated with survival, also in a larger validation set of 243 NSCLC patients. While these data are very encouraging, the investigators unfortunately measured these miRNAs in only one healthy donor, and their specificity for NSCLC is thus not sufficiently clear[52]. A comparison with more healthy controls was done with a pooled sample of 11 Chinese lung cancer patients, in whom 28 miRNAs were downregulated and 63 
miRNAs were upregulated compared to 11 male and 10 female normal controls. Two of the highest expressed miRNAs, miR-25 and miR-223, were validated in an independent set of 152 lung cancer sera and 75 normal sera and also found to be higher expressed in these patients[19].

A different approach was used by Silva et al, who preceded their tests by an EpCAM-based immunomagnetic enrichment step. Out of 365 candidates, no miRNAs were found to be upregulated in 28 patients as compared with 20 controls, but 10 miRNAs were downregulated. Three of these were differentially expressed in the validation step as well, and lower levels of let-7f were associated with shorter overall survival, while patients with lower levels of miR-30e-3p had shorter disease-free survival, without difference in overall survival[53].

\subsection{Prostate cancer}

In prostate cancer patients, a panel of six candidate miRNAs, selected on their expression in prostate tumors and lack of expression in healthy donor blood, was analyzed in two pools of 25 metastatic prostate cancer patients and 25 healthy controls, respectively. After out of the candidate miRNAs, miR-141 showed the greatest differential expression between the two pools, this miRNA was confirmed to be higher expressed in cancer patients on an individual level as well[21].

Brase and co-workers unfortunately did not validate their interesting findings of the upregulation of 5 miRNAs out of a panel of 667 candidate miRNAs in the serum of prostate cancer patients in healthy controls. They did observe that the expression of two of the 5 miRNAs, miR-375 and miR-141, was upregulated in malignant prostate tissue compared to benign prostate tissue, but concerns about the specificity of these miRNAs in serum remain[54].

Using an array method, Moltzahn and co-workers screened the expression level of 384 miRNAs in 12 healthy controls and 36 prostate cancer patients divided into three groups according to a validated risk score. The twelve miRNA candidates that were most differentially expressed between cancer patients and controls were validated by individual qRT-PCR, which confirmed the differential expression of nine miRNAs. No significant correlation was seen with risk scores or other clinicopathological parameters[55].

Lodes et al. used microarray profiling and found 15 miRNAs to be over-expressed in serum from 6 prostate cancer patients (all stage 3 and 4 ) relative to expression in 8 normal male controls[47].

\subsection{Ovarian cancer}

In ovarian cancer too, interest has been generated to detect miRNAs in the peripheral blood of cancer patients. Comparing 9 serum samples from ovarian cancer patients to 4 serum samples from healthy donors, 21 
differentially expressed miRNAs were identified. Eight could be confirmed in 19 cancer versus 11 normal specimens to be differentially expressed by RT-PCR, of which 5 (miR-21, -29a, -92 -126 and -29a) were upregulated expression in cancer patients, probably making these more suitable for clinical implementation[56]. MiRNA expression was also measured in EpCAM-positive exosomes. Exosomes are organelles that are actively released by tumors into the peripheral circulation and it was hypothesized that miRNAs detected in exosomes reflect those present in CTCs. Exosomes were isolated from 50 ovarian cancer patients and 20 controls using an immunomagnetic enrichment method based on anti-EpCAM. Eight miRNAs were found to be differentially expressed between the two groups[22].

\subsection{Gastric cancer}

Analyzing plasma samples of 69 gastric cancer patients taken before surgery and 30 healthy donors, five miRNAs were found to be differentially expressed[57]. Two of these five, miR-106a and miR-17, were also identified in samples of 90 patients (of which, remarkably, 49 were taken after resection of the primary tumor), to be differentially expressed compared to 27 healthy donors. Both miRNAs were expressed at a lower level after surgery compared to before surgery, but still differed about 10-fold from healthy controls[58].

As part of a larger study looking at liver pathology-specific miRNAs, gastric cancer patients were also evaluated for differential miRNA expression compared to controls. MiR-885-5p, which was also found to be upregulated in hepatocellular carcinoma patients (see below), was higher expressed in gastric cancer patients[59] compared to controls.

\subsection{Hepatocellular carcinoma}

MiR-500 was identified as highly expressed during fetal liver development and thus postulated to be involved in proliferation. Indeed, miR-500 was highly expressed in hepatocellular carcinoma cell lines, but its expression was higher in only 18 of 40 hepatocellular carcinomas compared to adjacent non-tumorous tissue, and in the serum of 3 of ten HCC patients[60].

Another study looking at HCC identified miR-885-5p as a miRNA of interest in this disease, being expressed higher in HCC patients than in healthy controls, liver cirrhosis and chronic hepatitis B patients[59].

\subsection{Colorectal cancer}

At least three studies have looked into the occurrence of selected candidate miRNAs in the plasma of colorectal cancer patients. A large study looked at samples from 120 primary colorectal cancer patients and 37 advanced adenoma patients, both taken before surgery, and compared them to 59 age-matched healthy controls who 
were confirmed to be without colorectal cancer by extensive diagnostic procedures including colonoscopy and CT scan. Two miRNAs, miR-29a and miR-92a, were identified from a training set and confirmed in the larger validation set to be upregulated in CRC plasma compared to controls. In adenoma patients too, these miRNAs were expressed higher than in controls, but significantly lower than in true cancer patients. Additionally, these two miRNAs decreased after surgery in another 20 colorectal cancer patients, suggesting that these miRNAs are in fact cancer-specific[61].

Another study also found MiR-92a to be higher expressed in CRC patients. Five miRNAs were selected based on higher expression in CRC plasma compared to healthy control plasma and higher expression in primary cancerous biopsies compared to adjacent non-cancerous colon tissue. Of these miRNAs, the two that were significantly elevated in $25 \mathrm{CRC}$ patients compared to controls and decreased after tumor resection (miR-92a and miR-17), were validated in an independent cohort of $90 \mathrm{CRC}$ patients and 50 controls. Additionally, both $\mathrm{miR}-92 a$ and $\mathrm{miR}-17$ were not expressed higher in patients with gastric cancer or inflammatory bowel disease, confirming their specificity[62].

Pu et al. chose to investigate miR-221 out of three miRNAs abundantly expressed in CRC, because of the good linearity in spiking samples obtained with this miRNA. In 103 CRC patients, miR-221 expression was higher than in 37 controls, however with a low specificity of $41 \%$ at the optimal cut-off level. MiR-221 expression did correlate with overall survival and p53 expression[63].

\subsection{Pancreatic cancer}

In pancreatic cancer, two miRNAs, miR-200a and miR-200b, involved in epithelial mesenchymal transition, were identified to be hypomethylated and overexpressed in primary tumors compared to surrounding normal pancreas tissue. In 45 serum samples obtained from pancreatic cancer patients before surgery, both miRNAs were expressed at a higher level than in samples from 32 healthy controls and 11 chronic pancreatitis patients[64].

Ho et al. looked for pancreatic cancer-specific expression of miR-210 in the circulation, as this miRNA increases under hypoxic conditions, which are known to correlate with poorer prognosis. miR-210 expression was measured in the plasma of a total of 22 locally advanced pancreatic cancer patients and 25 age-matched healthy controls, and confirmed to be 1.7-4-fold higher expressed in the patients[65].

\subsection{Head and neck cancer}


Wong et al. examined the expression of a large panel of miRNAs in tongue carcinomas and paired normal tissues, which identified 24 up regulated and 13 down regulated miRNAs. Because of its 59-fold higher expression in tumor tissue, miR-184 was further validated in an independent dataset and observed to be more abundant in plasma of patients with tongue squamous cell carcinoma than in controls. Additionally, miR-184 levels dropped after resection of the primary tumor[66].

\subsection{Esophageal squamous cell carcinoma (SCC)}

In esophageal SCC, one large study was recently published in which 25 miRNAs measured in serum were found to be upregulated in a pool of 141 cancer patients compared to controls. Of these 25,7 miRNAs were confirmed to be differentially expressed by individual QRT-PCR in a separate patient cohort, yielding higher AUCs than carcinoembryonic antigen (CEA)[67].

\subsection{Rhabdomyosarcoma}

Besides carcinomas, research has also been focused on specific miRNAs in rhabdomyosarcoma (RMS). Looking at RMS cell lines and primary tumor tissues, miR-206 was found to be most abundantly expressed among several muscle-specific miRNAs. MiR-206 was also the marker with the highest sensitivity and specificity in discriminating 10 RMS-patients from 28 patients with other pediatric tumor and 17 healthy donors, but miRs-1, 133a and 133b, involved in muscle proliferation and differentiation[68], were also higher expressed in RMS patients than in controls or non-RMS patients[69].

\section{CTC-associated miRNAs}

\subsection{The need for CTC-specific miRNAs detection}

As depicted above, studies on cell-free circulating miRNAs yield very interesting results and show the measurement of miRNAs in the circulation to be both feasible and clinically relevant. However, it is to be expected that not all miRNAs can actually be measured in the peripheral circulation. Especially in view of the fact that at least 100 different miRNAs already circulate in the blood of healthy donors [19,49], it is very likely that measuring these miRNAs in whole blood, serum or plasma from cancer patients can yield false-positive results. Several studies have identified circulating miRNAs that are differentially expressed between patients and healthy donors (Table 1). Most of these studies have measured miRNAs in the serum, plasma or exosome fractions of blood, instead of using whole blood. Using serum or plasma does for the most part eliminate the leukocyte background present in whole blood, but evidence has been presented that most miRNAs measured 
in these fractions are not actually derived from circulating epithelial cells[21]. Also, cellular miRNA expression patterns can differ from miRNA patterns released into the blood[70]. These studies raise the concern that cellfree miRNAs present in the circulation may not be a reliable representation of metastatic or primary tumor tissue, and that measuring CTC-associated miRNAs would be preferable. Besides possibly better representing the tumor load, measuring miRNAs in CTCs has the additional benefit of being able to correlate a miRNA signal to a CTC count, which aids in the interpretation of epithelial specificity.

\subsection{Enrichment of CTC-specific miRNAs}

When testing whether identified cell-free miRNAs can be measured in CTCs, or identifying new CTCassociated miRNAs, an enrichment step is pivotal. Most methods aimed at specifically molecularly characterizing CTCs in whole blood are preceded by such an enrichment step. Many methods are available, including enrichment based on size, density or specific marker expression[71]. These enrichment steps aim to isolate all CTCs from full blood, while getting rid of as many contaminating peripheral blood mononuclear cells (PBMCs) as possible. However, even when applying tumor-specific marker enrichment, hundreds to thousands of leukocytes are still present in the CTC-enriched fraction[12]. Also, the actual number of leukocytes may differ depending on tumor stage[50]. These leukocytes generate a background signal and thus complicate the measurement of CTC-specific miRNA expression, as only epithelial-specific miRNAs that are hardly expressed in leukocytes can be reliably measured. Many efforts are being made to develop a CTC isolation method that provides a purer CTC fraction for downstream analysis, based on for instance micromanipulation techniques[72]. Obtaining a higher purity of the enriched CTC fraction through more specific CTC isolation techniques would eliminate the need to only measure epithelial-specific genes, i.e., genes that are much higher expressed in CTCs than in leukocytes. So far, however, these techniques have not become widespread available and need further validation.

Despite these challenges, measuring CTC-associated has proven to be feasible. In our own work (manuscript submitted), we were able to identify 10 miRNAs more abundantly expressed in patients with $>5$ CTCs compared to patients without detectable CTCs and healthy donors.

\subsection{Remaining technical issues concerning the measurement of CTC specific miRNAs}


It is to be expected that the development of enrichment methods that provide a purer CTC fraction will simplify the measurement of CTC-associated miRNAs. In the meantime, a number of aspects need to be taken into consideration when measuring these CTC-specific miRNAs. Because of the low numbers of CTCs in the circulation, frequently less than 5 CTCs in $7.5 \mathrm{~mL}$ blood[73], sensitive RNA isolation techniques and unbiased pre-amplification steps are needed. Fortunately, kits are now on the market which enable the isolation of DNA, large RNA fragments (mRNA, >200 bp), small RNA fragments (micro- and non-coding RNA, <200 bp) and proteins in 4 separate aliquots from as little as one cell (Figure 2). After this sensitive fractionated RNA isolation, it is crucial that only epithelial-specific miRNAs are measured that are not or very weakly expressed in leukocytes. To estimate the ratio of the tumor cell-specific signal over leukocyte-derived signal, which is unfortunately present even after enrichment procedures, transcript levels of CTCs-specific miRNAs such as those in the miR-200/141 family[21,74] and leukocyte-specific miRNAs such as miR-429 can be compared[74]. The suitability of any miRNA combination to estimate epithelial-specific signal does however depends on the epithelial tumor cell type or subtype studied. Furthermore, due to the presence of cell-free EpCAM-positive exosomes in serum[22], it remains to be established which part of the miRNA signal from EpCAM-enriched CTC-fractions is actually derived from CTCs and which part from other EpCAM-enriched cells or cell fragments including exosomes. Another factor complicating miRNA measurement is the lack of an established constitutively expressed set of reference miRNAs that can be used to normalize candidate miRNA expression levels. MiR-16 has been used as a reference in several studies[23,50], but concerns have been raised due to its inconsistent expression in sera [47]. RNU/SNORs are also frequently used (Table 1), but it needs to be realized that these small RNA's are longer than the actual mature miRNAs being studied, making them less suitable for normalization. Furthermore, RNU6B (U6) has been reported to be degraded in serum samples[67]. Until consensus has been established on a robust reference miRNA set, normalizing on the mean expression of all expressed and CTC-specific miRNAs is probably the optimal method when multiple miRNA transcripts are measured at the same time[75]. In Figure 2 we summarize the steps that need to be taken to ensure epithelial tumor cell specific gene expression profiling of CTCs.

\section{Concluding remarks}

When critically looking at the data generated thus far measuring miRNA expression in the circulation, a few remarks must be made. 
Firstly, the methods that are used to identify differentially expressed miRNAs vary greatly; many researchers start off with candidate miRNAs of interest that have previously been associated with a cancer type, while others look at all differentially expressed miRNAs between healthy controls and patients or between tumor tissue and normal adjacent tissue. This latter approach also enables the identification of potential up- or downmodulated pathways associated with differentially expressed miRNA transcripts. Furthermore, a combination of several higher and lower expressed miRNAs is likely more informative than analysis of the expression of a single marker alone.

Secondly, some studies have unfortunately failed to validate their results in healthy controls, raising concerns about the specificity of potentially interesting miRNAs. Similarly, as with CTC enumeration, and also with regard to gene expression profiling in CTCs, specificity is pivotal when trying to identify tumor-specific signal particularly when contaminating leukocytes are present.

Lastly, any miRNA that is identified in a patient cohort to be differentially expressed or associated with prognosis should be validated in an independent cohort and reach acceptable sensitivity and specificity before it can be implemented into the clinic for routine analysis. While some studies have used validation sets, most have not, hampering the translation from bench to bedside.

Despite these possible flaws in some studies, measuring circulating miRNAs, cell-free or CTC associated, has proven to be feasible, can generate tumor-specific results, and may thus be of clinical relevance if their expression can be robustly measured and is sufficiently correlated with clinical outcome parameters such as overall survival or therapy response. The value of circulating miRNAs is expected to increase rapidly with the development of techniques that are able to isolate a more pure CTC fraction. Such an improvement will enable the measurement of any of the discussed prognostic and predictive miRNAs that have been identified in primary tumor tissue. Even more so than prognostic factors, the oncology field is devoid of reliable and robust predictive factors that adequately guide oncologists in the choice of optimal treatment for their patients. MiRNAs in the circulation may provide a new opportunity in this direction because of their stability and far-stretched effects in cancer biology and disease progression, and research should be aimed towards identifying and validating the predictive potential of these markers.

MiRNAs are a valuable addition to the information that CTCs already provide. In Figure $\mathbf{3}$ we have depicted our view of the future role of CTC analysis before and during treatment of cancer patients, providing both prognostic, predictive and drug target information at different time points. 


\section{Expert commentary}

MiRNAs have become an important research field, and have proven their value as regulators in carcinogenesis and cancer progression in many different cancer types. If these relatively stable small RNAs can be robustly detected by highly sensitive PCR methods routinely available in most laboratories, they can become a new class of biomarkers. More important for the clinical setting, the prognostic value of certain miRNAs has been established in various cancer types and data are slowly emerging on their predictive value. These clinically relevant miRNAs are subsequently sought for in the circulation of cancer patients to enable their repeated and non-invasive measurement. A few issues have to be accounted for before miRNA expression in the circulation as a tool to predict prognosis or therapy response is ready for the clinic.

Firstly, consensus should be established on which fraction of peripheral blood should be used to measure miRNAs. Current studies have been performed in non-enriched or enriched whole blood, serum and plasma, without clear data being available on the distribution of miRNAs is these different blood compartments. It is conceivable that only a selection of miRNAs is, actively or passively, shed from circulating tumor cells. Also, the use of an enrichment marker for whole blood can greatly influence the amount and type of CTCs that are subsequently characterized.

Secondly, more data should be generated on the occurrence and expression levels of circulating miRNAs in healthy individuals. This can be done either by testing selected panels of miRNAs in a large cohort of genderand otherwise matched healthy controls in parallel with cancer patients.

Thirdly, the discussion on which constitutively expressed miRNAs to use as a reference gene set is ongoing and it remains to be seen if a cell type-independent panel can be identified. Until that time, it is imperative that each study clearly states their normalization method and their reasons to choose said method.

\section{Five-year view}

While enumeration of CTCs has already proven its strength, the future of molecular analysis lies in the development of CTC isolation assays that generate higher CTC numbers and thus increased tumor DNA, mRNA and miRNA content, and reduce the number of contaminating leukocytes. We have already shown that the enrichment marker used in one of the most common CTC detection assays, the EpCAM-based CellSearch technique, is not expressed in all breast cancer subtypes[76]. This epithelial cell adhesion molecule (EpCAM) 
does not detect normal-like breast cancer cells, which can however be detected by adding CD146 in the enrichment step[77]. This is just one example of a probably more widespread problem of marker heterogeneity among cancer subtypes, which causes us to miss a subset of CTCs. The development of a method that either uses a panel of antibodies to detect CTCs, or a method that is independent of marker expression, but instead on for example physical properties of tumor cells, is probably the answer to this problem. Especially the latter option, selecting CTCs based on unique properties such as membrane stiffness, is a still developing field[78]. Following this selection step by micromanipulation will enable the isolation of single CTCs, which makes them available for downstream applications such as whole genome DNA or transcriptome sequencing, but also allowing culturing of these cells.

In a few years time, anti-miRNA treatments will probably become available, and it is very likely that at least some of these miRNAs will be detectable in the circulation. This could simplify and fasten the testing of these drugs in phase I and II clinical trials, as the level of the target miRNAs can be directly measured in the patients' blood.

\section{Key issues}

- There is an urgent need for additional diagnostic, prognostic and predictive markers in oncology

- These markers should preferably be measurable at any time during the course of the disease

- Circulating tumor cells provide an unique opportunity to diagnose the origin and type of primary tumor, and to assess prognosis, response to therapy and drug targets non-invasively and repeatedly

- Among these markers, miRNAs are especially promising because of their stability and pivotal regulatory role in carcinogenesis

- Many miRNAs have already been identified to be of prognostic value in primary tumors

- Some miRNAs have shown predictive value in cell lines and patients

- Measuring miRNAs in the circulation is feasible and, depending on the choice of miRNA, can be cancerspecific

- A properly sized control cohort of healthy blood donors is a pre-requisite for these kind of studies

- So far, very few circulating tumor cell associated miRNAs have been associated with prognosis or therapy response 
- The technical challenge is to discriminate between epithelial tumor cell-specific miRNAs and miRNAs from background leukocytes 
1. Cristofanilli $\mathrm{M}$, Budd $\mathrm{Gt}$, Ellis $\mathrm{Mj}$ et al. Circulating tumor cells, disease progression, and survival in metastatic breast cancer. The New England journal of medicine 351(8), 781-791 (2004).

2. Cristofanilli M, Hayes Df, Budd Gt et al. Circulating tumor cells: a novel prognostic factor for newly diagnosed metastatic breast cancer. J Clin Oncol 23(7), 1420-1430 (2005).

3. Cohen Sj, Punt Cj, lannotti N et al. Relationship of circulating tumor cells to tumor response, progression-free survival, and overall survival in patients with metastatic colorectal cancer. $J$ Clin Oncol 26(19), 3213-3221 (2008).

4. De Bono Js, Scher Hi, Montgomery $\mathrm{Rb}$ et al. Circulating tumor cells predict survival benefit from treatment in metastatic castration-resistant prostate cancer. Clin Cancer Res 14(19), 6302-6309 (2008).

5. Hayes Df, Cristofanilli M, Budd Gt et al. Circulating tumor cells at each follow-up time point during therapy of metastatic breast cancer patients predict progression-free and overall survival. Clin Cancer Res 12(14 Pt 1), 4218-4224 (2006).

6. Attard G, Swennenhuis Jf, Olmos D et al. Characterization of ERG, AR and PTEN gene status in circulating tumor cells from patients with castration-resistant prostate cancer. Cancer Res 69(7), 2912-2918 (2009).

7. Leversha Ma, Han J, Asgari Z et al. Fluorescence in situ hybridization analysis of circulating tumor cells in metastatic prostate cancer. Clin Cancer Res 15(6), 2091-2097 (2009).

8. Pestrin M, Bessi S, Galardi F et al. Correlation of HER2 status between primary tumors and corresponding circulating tumor cells in advanced breast cancer patients. Breast cancer research and treatment (2009).

9. Jiang Y, Palma Jf, Agus Db, Wang Y, Gross Me. Detection of androgen receptor mutations in circulating tumor cells in castration-resistant prostate cancer. Clin Chem 56(9), 1492-1495 (2010).

10. Maheswaran S, Sequist Lv, Nagrath $S$ et al. Detection of mutations in EGFR in circulating lung-cancer cells. The New England journal of medicine 359(4), 366-377 (2008).

11. Munzone E Nf, Zorzino L, Medici M, Minchella I, Cassatella Mc, Casadio C, Picozzi M, Adamoli L, Goldhirsch a, Sandri M. Acquisition of HER2/neu over-expression on circulating tumor cells (CTCs) in patients (pts) with advanced breast cancer (ABC) during chemotherapy. J Clin Oncol 26(May 20 Suppl; abstr 11017)(2008).

12. Sieuwerts Am, Kraan J, Bolt-De Vries J et al. Molecular characterization of circulating tumor cells in large quantities of contaminating leukocytes by a multiplex real-time PCR. Breast cancer research and treatment 118(3), 455-468 (2009).

13. Aktas B, Tewes M, Fehm T, Hauch S, Kimmig R, Kasimir-Bauer S. Stem cell and epithelialmesenchymal transition markers are frequently over-expressed in circulating tumor cells of metastatic breast cancer patients. Breast Cancer Res 11(4), R46 (2009).

14. Fehm T, Muller V, Aktas B et al. HER2 status of circulating tumor cells in patients with metastatic breast cancer: a prospective, multicenter trial. Breast cancer research and treatment 124(2), 403-412 (2010).

15. Lee Rc, Feinbaum RI, Ambros V. The C. elegans heterochronic gene lin-4 encodes small RNAs with antisense complementarity to lin-14. Cell 75(5), 843-854 (1993).

16. Mattick Js, Makunin Iv. Non-coding RNA. Human molecular genetics 15 Spec No 1, R17-29 (2006).

17. Calin Ga, Croce Cm. MicroRNA signatures in human cancers. Nat Rev Cancer 6(11), 857866 (2006).

18. Wiemer Ea. The role of microRNAs in cancer: no small matter. Eur J Cancer 43(10), 15291544 (2007).

19. Chen $\mathrm{X}, \mathrm{Ba} \mathrm{Y}, \mathrm{Ma} \mathrm{L}$ et al. Characterization of microRNAs in serum: a novel class of biomarkers for diagnosis of cancer and other diseases. Cell research 18(10), 997-1006 (2008).

20. Gilad S, Meiri E, Yogev $\mathrm{Y}$ et al. Serum microRNAs are promising novel biomarkers. PLoS ONE 3(9), e3148 (2008). 
21. Mitchell Ps, Parkin Rk, Kroh Em et al. Circulating microRNAs as stable blood-based markers for cancer detection. Proc Natl Acad Sci U S A 105(30), 10513-10518 (2008).

22. Taylor Dd, Gercel-Taylor C. MicroRNA signatures of tumor-derived exosomes as diagnostic biomarkers of ovarian cancer. Gynecologic oncology 110(1), 13-21 (2008).

23. Asaga S, Kuo C, Nguyen T, Terpenning M, Giuliano Ae, Hoon Ds. Direct Serum Assay for microRNA-21 Concentrations in Early and Advanced Breast Cancer. Clin Chem, Oct 29 [ Epub ahead of print] (2010).

24. Brase Jc, Wuttig D, Kuner R, Sultmann H. Serum microRNAs as non-invasive biomarkers for cancer. Molecular cancer 9(1), 306 (2010).

25. Li J, Smyth P, Flavin R et al. Comparison of miRNA expression patterns using total RNA extracted from matched samples of formalin-fixed paraffin-embedded (FFPE) cells and snap frozen cells. BMC biotechnology 7, 36 (2007).

26. Kim Vn. MicroRNA biogenesis: coordinated cropping and dicing. Nature reviews 6(5), 376385 (2005).

27. Vasudevan S, Tong Y, Steitz Ja. Switching from repression to activation: microRNAs can upregulate translation. Science (New York, N.Y 318(5858), 1931-1934 (2007).

28. Miranda Kc, Huynh T, Tay $Y$ et al. A pattern-based method for the identification of MicroRNA binding sites and their corresponding heteroduplexes. Cell 126(6), 1203-1217 (2006).

29. Bartel Dp. MicroRNAs: genomics, biogenesis, mechanism, and function. Cell 116(2), 281-297 (2004).

30. Lu J, Getz G, Miska Ea et al. MicroRNA expression profiles classify human cancers. Nature 435(7043), 834-838 (2005).

31. Rosenfeld N, Aharonov R, Meiri E et al. MicroRNAs accurately identify cancer tissue origin. Nature biotechnology 26(4), 462-469 (2008).

32. Rosenwald S, Gilad S, Benjamin S et al. Validation of a microRNA-based qRT-PCR test for accurate identification of tumor tissue origin. Mod Pathol 23(6), 814-823 (2010).

33. Sempere Lf, Christensen M, Silahtaroglu A et al. Altered MicroRNA expression confined to specific epithelial cell subpopulations in breast cancer. Cancer Res 67(24), 11612-11620 (2007).

34. Ferracin M, Veronese A, Negrini M. Micromarkers: miRNAs in cancer diagnosis and prognosis. Expert review of molecular diagnostics 10(3), 297-308 (2010).

35. Yang $\mathrm{H}$, Kong $\mathrm{W}, \mathrm{He} \mathrm{L}$ et al. MicroRNA expression profiling in human ovarian cancer: miR214 induces cell survival and cisplatin resistance by targeting PTEN. Cancer Res 68(2), 425433 (2008).

36. Weiss $\mathrm{Gj}$, Bemis Lt, Nakajima $\mathrm{E}$ et al. EGFR regulation by microRNA in lung cancer: correlation with clinical response and survival to gefitinib and EGFR expression in cell lines. Ann Oncol 19(6), 1053-1059 (2008).

37. Ji J, Shi J, Budhu A et al. MicroRNA expression, survival, and response to interferon in liver cancer. The New England journal of medicine 361(15), 1437-1447 (2009).

38. Salter Kh, Acharya $\mathrm{Cr}$, Walters $\mathrm{Ks}$ et al. An integrated approach to the prediction of chemotherapeutic response in patients with breast cancer. PLoS ONE 3(4), e1908 (2008).

39. Blower Pe, Chung Jh, Verducci Js et al. MicroRNAs modulate the chemosensitivity of tumor cells. Molecular cancer therapeutics 7(1), 1-9 (2008).

40. Rodriguez-Gonzalez Fg, Sieuwerts Am, Smid M et al. MicroRNA-30c expression level is an independent predictor of clinical benefit of endocrine therapy in advanced estrogen receptor positive breast cancer. Breast cancer research and treatment, May 19 [Epub ahead of print] (2010).

41. Si Ml, Zhu S, Wu H, Lu Z, Wu F, Mo Yy. miR-21-mediated tumor growth. Oncogene 26(19), 2799-2803 (2007).

42. Meng $\mathrm{F}$, Henson R, Lang $\mathrm{M}$ et al. Involvement of human micro-RNA in growth and response to chemotherapy in human cholangiocarcinoma cell lines. Gastroenterology 130(7), 21132129 (2006). 
43. Iorio Mv, Casalini P, Piovan C et al. microRNA-205 regulates HER3 in human breast cancer. Cancer Res 69(6), 2195-2200 (2009).

44. Kota J, Chivukula $\mathrm{Rr}$, O'donnell $\mathrm{Ka}$ et al. Therapeutic microRNA delivery suppresses tumorigenesis in a murine liver cancer model. Cell 137(6), 1005-1017 (2009).

45. Krutzfeldt J, Rajewsky N, Braich R et al. Silencing of microRNAs in vivo with 'antagomirs'. Nature 438(7068), 685-689 (2005).

46. Fabbri $\mathrm{M}$, Croce $\mathrm{Cm}$, Calin $\mathrm{Ga}$. MicroRNAs in the ontogeny of leukemias and lymphomas. Leukemia \& lymphoma 50(2), 160-170 (2009).

47. Lodes Mj, Caraballo M, Suciu D, Munro S, Kumar A, Anderson B. Detection of cancer with serum miRNAs on an oligonucleotide microarray. PLoS ONE 4(7), e6229 (2009).

48. Heneghan Hm, Miller N, Lowery Aj, Sweeney Kj, Newell J, Kerin Mj. Circulating microRNAs as novel minimally invasive biomarkers for breast cancer. Annals of surgery 251(3), 499-505 (2010).

49. Zhao H, Shen J, Medico L, Wang D, Ambrosone Cb, Liu S. A pilot study of circulating miRNAs as potential biomarkers of early stage breast cancer. PLoS ONE 5(10), e13735 (2010).

50. Roth C, Rack B, Muller V, Janni W, Pantel K, Schwarzenbach H. Circulating microRNAs as blood-based markers for patients with primary and metastatic breast cancer. Breast Cancer Res 12(6), R90 (2010).

51. Zhu W, Qin W, Atasoy U, Sauter Er. Circulating microRNAs in breast cancer and healthy subjects. BMC research notes 2, 89 (2009).

52. Hu Z, Chen $X$, Zhao $Y$ et al. Serum microRNA signatures identified in a genome-wide serum microRNA expression profiling predict survival of non-small-cell lung cancer. $J$ Clin Oncol 28(10), 1721-1726 (2010).

53. Silva J, Garcia V, Zaballos A et al. Vesicle-related microRNAs in plasma of NSCLC patients and correlation with survival. Eur Respir J (2010).

54. Brase Jc, Johannes M, Schlomm $\mathrm{T}$ et al. Circulating miRNAs are correlated with tumor progression in prostate cancer. Int J Cancer, Apr 5 [Epub ahead of print] (2010).

55. Moltzahn F, Olshen Ab, Baehner $L$ et al. Microfluidic based multiplex qRT-PCR identifies diagnostic and prognostic microRNA signatures in sera of prostate cancer patients. Cancer Res, Nov 22 [Epub ahead of print] (2010).

56. Resnick Ke, Alder $\mathrm{H}$, Hagan Jp, Richardson DI, Croce $\mathrm{Cm}$, Cohn De. The detection of differentially expressed microRNAs from the serum of ovarian cancer patients using a novel real-time PCR platform. Gynecologic oncology 112(1), 55-59 (2009).

57. Tsujiura M, Ichikawa D, Komatsu $S$ et al. Circulating microRNAs in plasma of patients with gastric cancers. Br J Cancer 102(7), 1174-1179 (2010).

58. Zhou H, Guo Jm, Lou Yr et al. Detection of circulating tumor cells in peripheral blood from patients with gastric cancer using microRNA as a marker. Journal of molecular medicine (Berlin, Germany) 88(7), 709-717 (2010).

59. Gui J, Tian $Y$, Wen $X$ et al. Serum microRNA characterization identifies miR-885-5p as a potential marker for detecting liver pathologies. Clin Sci (Lond) (2010).

60. Yamamoto $\mathrm{Y}$, Kosaka N, Tanaka M et al. MicroRNA-500 as a potential diagnostic marker for hepatocellular carcinoma. Biomarkers 14(7), 529-538 (2009).

61. Huang Z, Huang D, Ni S, Peng Z, Sheng W, Du X. Plasma microRNAs are promising novel biomarkers for early detection of colorectal cancer. Int J Cancer 127(1), 118-126 (2010).

62. Ng Ek, Chong Ww, Jin $\mathrm{H}$ et al. Differential expression of microRNAs in plasma of patients with colorectal cancer: a potential marker for colorectal cancer screening. Gut 58(10), 1375-1381 (2009).

63. $\mathrm{Pu} \mathrm{Xx}$, Huang $\mathrm{Gl}$, Guo $\mathrm{Hq}$ et al. Circulating miR-221 directly amplified from plasma is a potential diagnostic and prognostic marker of colorectal cancer and is correlated with p53 expression. Journal of gastroenterology and hepatology 25(10), 1674-1680 (2010). 
64. Li A, Omura N, Hong Sm et al. Pancreatic cancers epigenetically silence SIP1 and hypomethylate and overexpress miR-200a/200b in association with elevated circulating miR200a and miR-200b levels. Cancer Res 70(13), 5226-5237 (2010).

65. Ho As, Huang X, Cao $\mathrm{H}$ et al. Circulating miR-210 as a Novel Hypoxia Marker in Pancreatic Cancer. Translational oncology 3(2), 109-113 (2010).

66. Wong Ts, Liu Xb, Wong By, Ng Rw, Yuen Ap, Wei Wi. Mature miR-184 as Potential Oncogenic microRNA of Squamous Cell Carcinoma of Tongue. Clin Cancer Res 14(9), 25882592 (2008).

67. Zhang $\mathrm{C}$, Wang $\mathrm{C}$, Chen $\mathrm{X}$ et al. Expression Profile of MicroRNAs in Serum: A Fingerprint for Esophageal Squamous Cell Carcinoma. Clin Chem 56(12), 1871-1879 (2010).

68. Chen Jf, Mandel Em, Thomson Jm et al. The role of microRNA-1 and microRNA-133 in skeletal muscle proliferation and differentiation. Nature genetics 38(2), 228-233 (2006).

69. Miyachi M, Tsuchiya K, Yoshida $\mathrm{H}$ et al. Circulating muscle-specific microRNA, miR-206, as a potential diagnostic marker for rhabdomyosarcoma. Biochemical and biophysical research communications 400(1), 89-93 (2010).

70. Pigati L, Yaddanapudi Sc, lyengar $\mathrm{R}$ et al. Selective release of microRNA species from normal and malignant mammary epithelial cells. PLoS ONE 5(10), e13515 (2010).

71. Mostert B, Sleijfer S, Foekens Ja, Gratama Jw. Circulating tumor cells (CTCs): detection methods and their clinical relevance in breast cancer. Cancer treatment reviews 35(5), 463474 (2009).

72. Breton $\mathrm{F}$, Bennetau $\mathrm{B}$, Lidereau $\mathrm{R}$ et al. A mesofluidic multiplex immunosensor for detection of circulating cytokeratin-positive cells in the blood of breast cancer patients. Biomedical microdevices, Sep 3 [Epub ahead of print] (2010).

73. Allard Wj, Matera J, Miller Mc et al. Tumor cells circulate in the peripheral blood of all major carcinomas but not in healthy subjects or patients with nonmalignant diseases. Clin Cancer Res 10(20), 6897-6904 (2004).

74. Lin Z, Wang X, Fewell C, Cameron J, Yin Q, Flemington Ek. Differential expression of the miR-200 family microRNAs in epithelial and B cells and regulation of Epstein-Barr virus reactivation by the miR-200 family member miR-429. Journal of virology 84(15), 7892-7897 (2010).

75. Mestdagh $\mathrm{P}$, Van Vlierberghe $\mathrm{P}$, De Weer $\mathrm{A}$ et al. A novel and universal method for microRNA RT-qPCR data normalization. Genome biology 10(6), R64 (2009).

76. Sieuwerts Am, Kraan J, Bolt J et al. Anti-epithelial cell adhesion molecule antibodies and the detection of circulating normal-like breast tumor cells. Journal of the National Cancer Institute 101(1), 61-66 (2009).

77. Mostert B, Kraan J, Bolt-De Vries J et al. Detection of circulating tumor cells in breast cancer may improve through enrichment with anti-CD146. Breast cancer research and treatment, Apr 9 [Epub ahead of print] (2010).

78. Gascoyne Pr, Noshari J, Anderson Tj, Becker Ff. Isolation of rare cells from cell mixtures by dielectrophoresis. Electrophoresis 30(8), 1388-1398 (2009).

79. Crosby Me, Kulshreshtha R, Ivan M, Glazer Pm. MicroRNA regulation of DNA repair gene expression in hypoxic stress. Cancer Res 69(3), 1221-1229 (2009).

80. Foekens Ja, Sieuwerts Am, Smid M et al. Four miRNAs associated with aggressiveness of lymph node-negative, estrogen receptor-positive human breast cancer. Proc Natl Acad Sci U $S$ A 105(35), 13021-13026 (2008).

81. Giannakakis A, Sandaltzopoulos R, Greshock J et al. miR-210 links hypoxia with cell cycle regulation and is deleted in human epithelial ovarian cancer. Cancer biology \& therapy 7(2), 255-264 (2008).

82. Kim Hw, Haider Hk, Jiang S, Ashraf M. Ischemic preconditioning augments survival of stem cells via miR-210 expression by targeting caspase-8-associated protein 2 . The Journal of biological chemistry 284(48), 33161-33168 (2009).

83. Mizuno $\mathrm{Y}$, Tokuzawa $\mathrm{Y}$, Ninomiya $\mathrm{Y}$ et al. miR-210 promotes osteoblastic differentiation through inhibition of AcvR1b. FEBS letters 583(13), 2263-2268 (2009). 
84. Pulkkinen K, Malm T, Turunen M, Koistinaho J, Yla-Herttuala S. Hypoxia induces microRNA miR-210 in vitro and in vivo ephrin-A3 and neuronal pentraxin 1 are potentially regulated by miR-210. FEBS letters 582(16), 2397-2401 (2008).

85. Zhang Z, Sun $\mathrm{H}$, Dai $\mathrm{H}$ et al. MicroRNA miR-210 modulates cellular response to hypoxia through the MYC antagonist MNT. Cell cycle (Georgetown, Tex 8(17), 2756-2768 (2009).

86. Huang $\mathrm{X}$, Ding $\mathrm{L}$, Bennewith $\mathrm{KI}$ et al. Hypoxia-inducible mir-210 regulates normoxic gene expression involved in tumor initiation. Molecular cell 35(6), 856-867 (2009).

87. Huang X, Le Qt, Giaccia Aj. MiR-210--micromanager of the hypoxia pathway. Trends in molecular medicine 16(5), 230-237 (2010).

88. Fasanaro P, Greco S, Lorenzi $\mathrm{M}$ et al. An integrated approach for experimental target identification of hypoxia-induced miR-210. The Journal of biological chemistry 284(50), 35134-35143 (2009). 
Figure 1: miRNA processing resulting in altered protein expression

\section{Left panel: miRNA processing}

In the nucleus, miRNAs are transcribed into primary miRNA transcripts (pri-miRNAs). These pri-miRNAs are subsequently cleaved into precursor miRNAs (pre-miRNAs) by Drosha. These pre-miRNAs, 70 to 90 nucleotides in length, are transported into the cytoplasm by Exportin 5 where the hairpin precursors are cleaved by Dicer, resulting in a small dsRNA duplex that contains both the mature miRNA strand and its complementary strand. The 20 to 25 nucleotides in length mature miRNA strand is then incorporated into a RNA-induced silencing complex (RISC), which inhibits the function of its target mRNA by mRNA degradation or, most commonly, by translational repression[26,79]. (Figure freely adapted from http://www.marligen.com)

Right panel: an example of the multiple functions of miR-210 in the for cancer important process of hypoxia, which allows cancer cells to adapt to a low oxygen environment

Increased miR-210 expression has been linked to increased metastatic capability and increased hypoxia signaling in lymph node-negative estrogen receptor-positive human breast cancer[80]. MiR-210 is regulated by hypoxia-inducible factor 1-alpha (HIF-1alpha), and the expression of both is increased in response to hypoxia. By up regulating miR-210 expression during hypoxia, resulting in the repressed translation of its multiple target genes[79,81-88] and direct or indirect down and up regulation of downstream proteins[88], cells can adjust and adapt to an hypoxic environment. 
Figure 2: Step-by-step scheme for reliable measurement of CTC-associated miRNAs

After collecting blood samples in EDTA tubes to preserve RNA integrity and enrichment for CTCs (1), a sensitive isolation technique - preferably one that is able to isolate genomic DNA, mRNA, miRNA and protein in separate fractions (2) - and linear pre-amplification steps (3) are needed to enable detection of molecules in material from as little as one cell.

Next, CTC- and leukocyte-specific signals are used to estimate the ratio of the tumor cell-specific signal over leukocyte-derived signal (4). Now, samples can be grouped into those with detectable epithelial-derived signals (5a) and those without detectable epithelial signals (5b), with the latter group comprising both patient samples without detectable epithelial signals and samples from healthy donors. Until consensus has been established on a robust reference miRNA set, normalizing on the mean expression of all expressed miRNAs in both groups (6a and $\mathbf{6 b})$ is probably the optimal method when multiple miRNA transcripts are measured at the same time.

Finally, to ensure epithelial tumor cell-specific gene expression profiling of CTCs, levels measured in the samples without detectable epithelial signals $(\mathbf{6 b})$ are used as cut-off for the samples with detectable epithelial signals $(6 a)$ to calculate the remaining CTC-specific signals (7). 
Figure 3: implementation of CTC enumeration and characterization into the clinic

Here, we explain how we envision the place of CTC enumeration and characterization in the cancer management of the future. Before starting a new line of systemic treatment for metastatic cancer patients, CTC enumeration and isolation can be performed. A CTC count will clarify the prognosis of the patient, and an up-todate characterization of gDNA mutations and methylation, mRNA and miRNA expression and receptor status helps the clinician to select the most effective tumor therapy. After the first cycle of chemotherapy, CTC enumeration and isolation will be repeated; a rise in CTC count can be an argument to switch therapy, as is a switch in receptor status, acquired or loss of mutations and altered mRNA or miRNA expression. Drug targets, predictive and prognostic factors can be continuously reassessed during the course of treatment and at the time of relapse in a non-invasive manner, resulting in truly patient-tailored treatment. 
Table 1: Circulating cell-free miRNAs

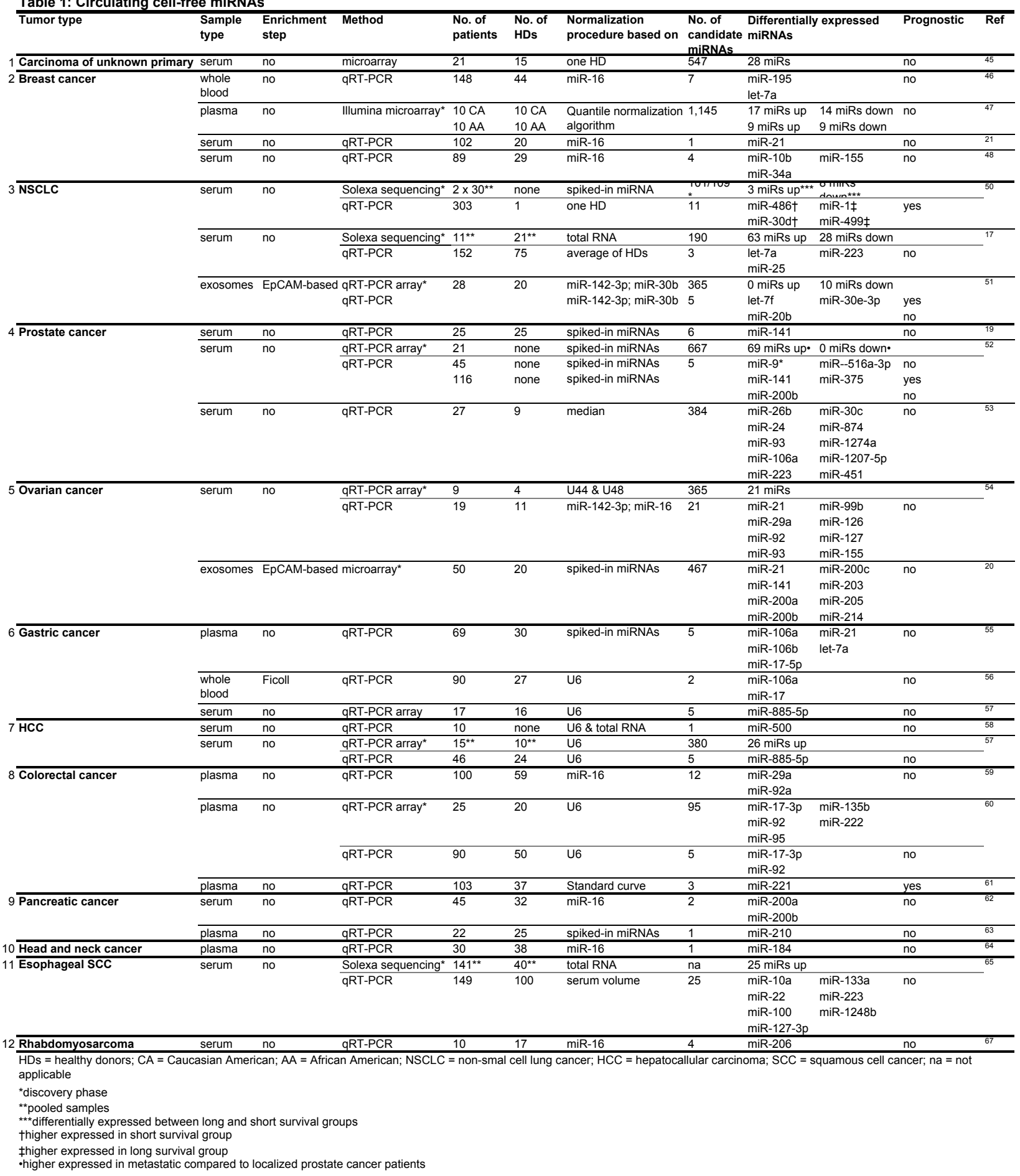




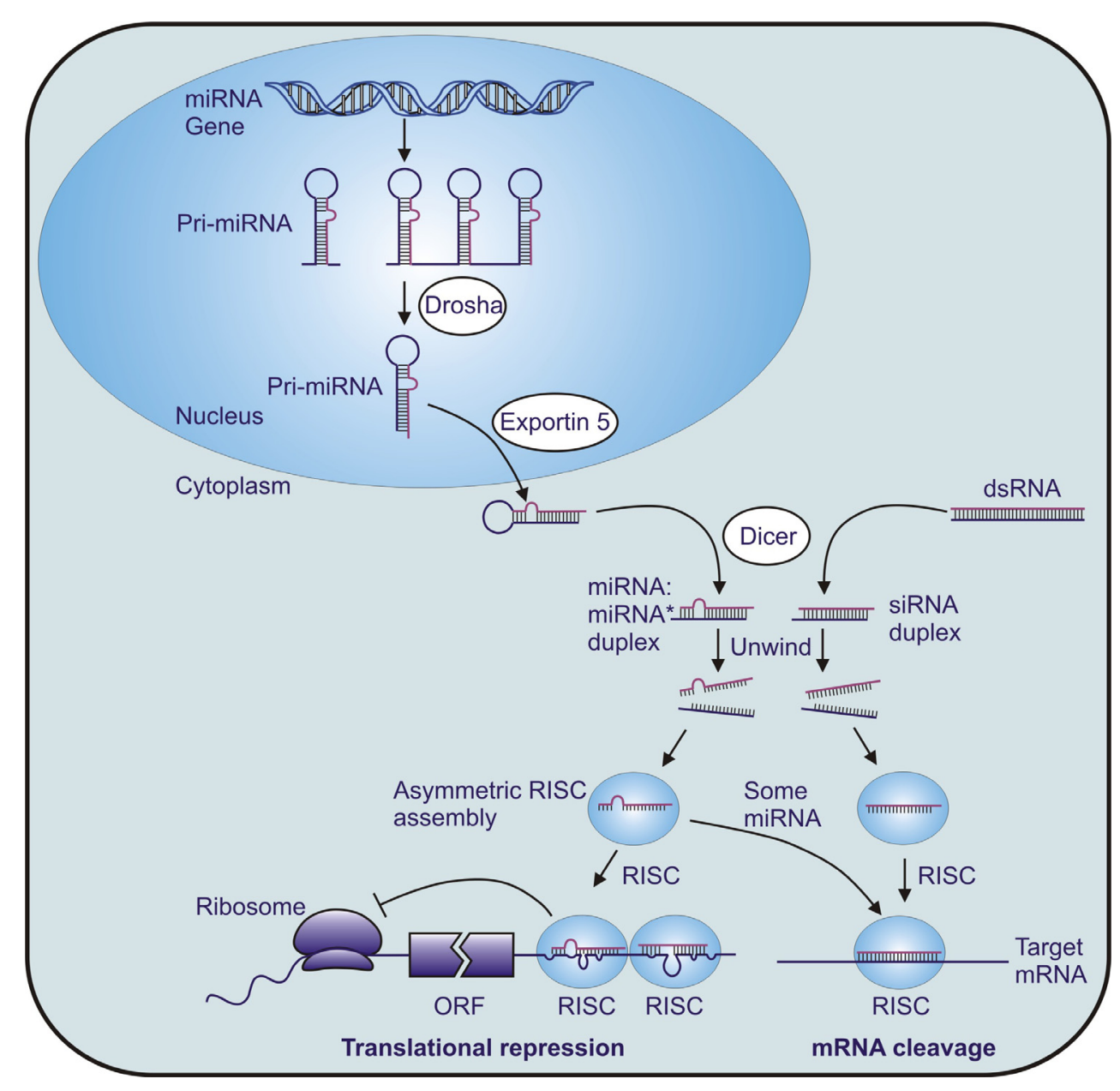

\section{HYPOXIA}

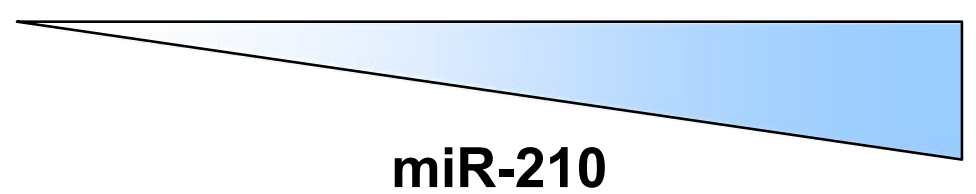

\section{Examples of miR-210 targets}

\begin{tabular}{|lll|}
\hline ACRV1B & ISCU & MNT \\
CASP8AP2 & NPTX1 & CDK10 \\
E2F3 & EFNA3 & APC \\
FGFRL1 & HOXA1/9 & RAD51/52 \\
\hline
\end{tabular}

Examples of proteins directly or indirectly modulated by miR-210

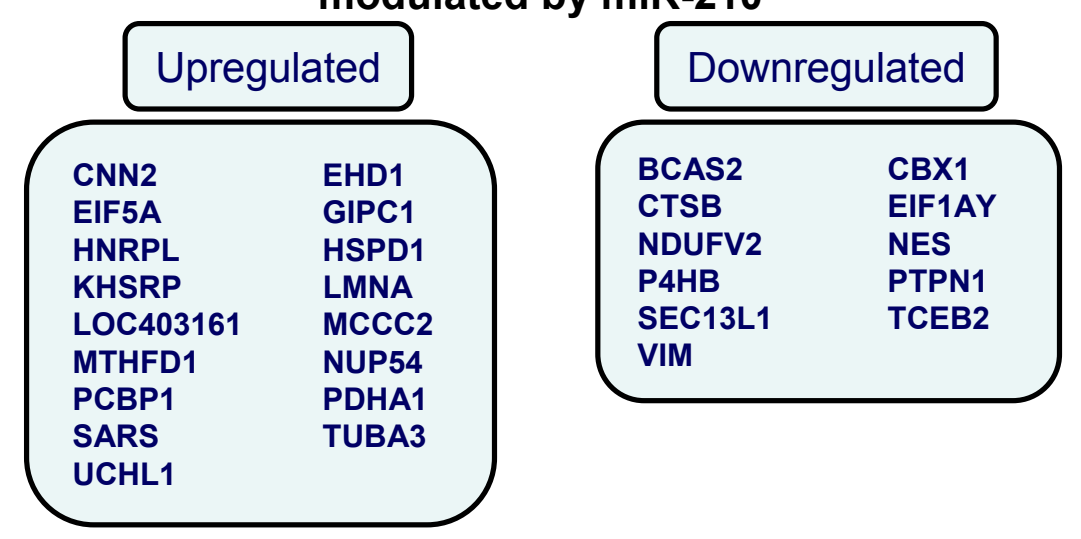




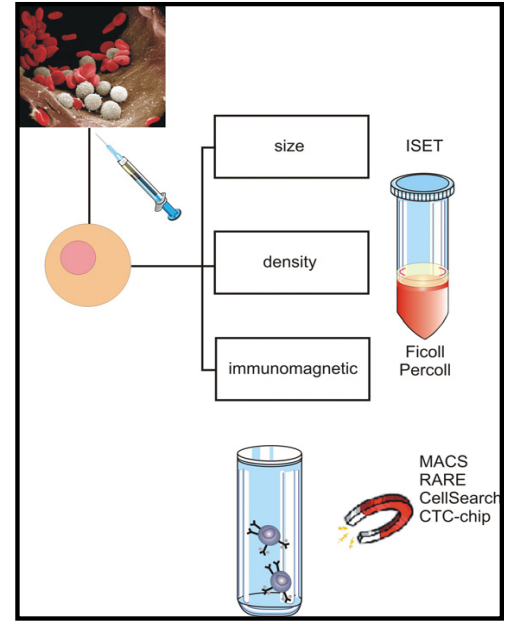

2

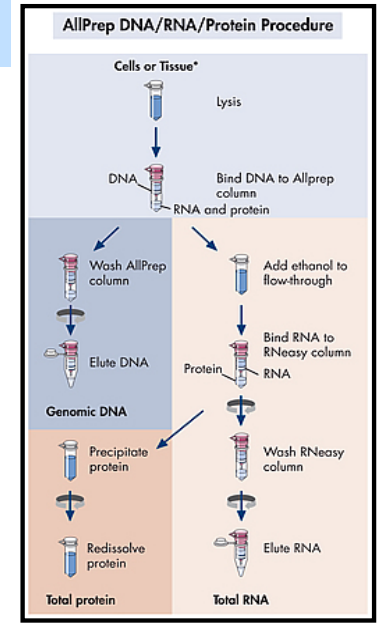

4

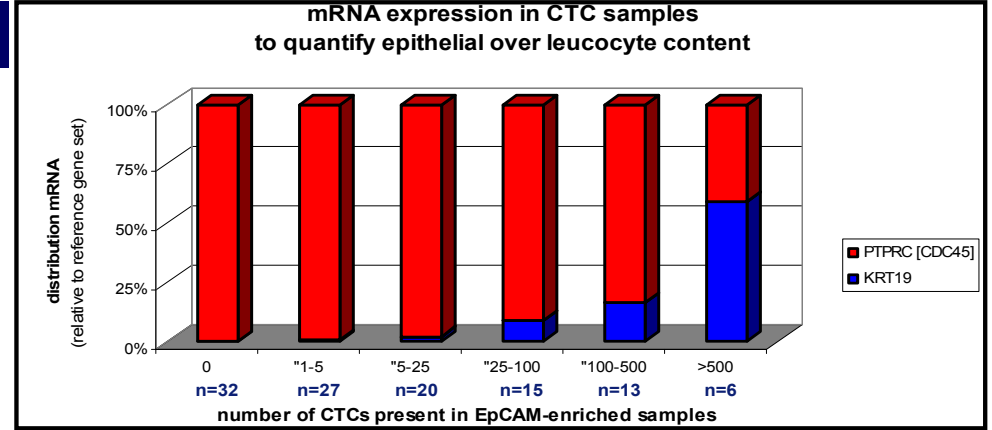

7

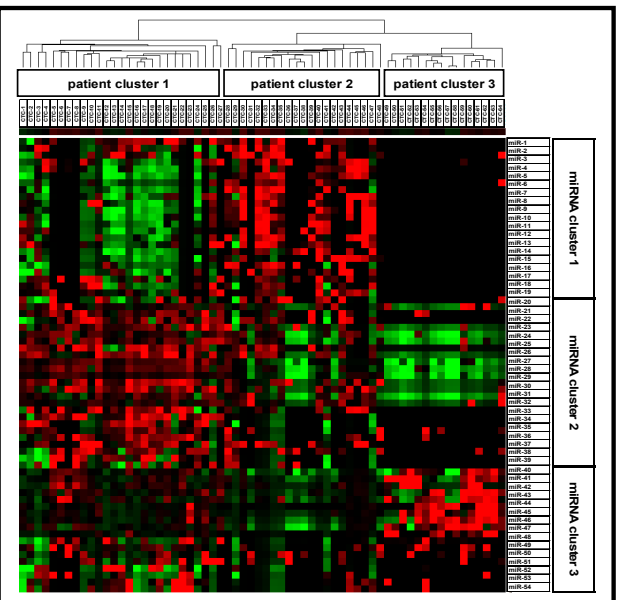

1 Collect blood sample and enrich for CTCs

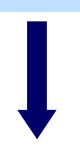

2 Extract mRNA, miRNA, genomic DNA and protein separately

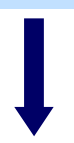

3 Linear pre-amplification

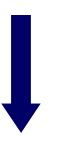

4 Estimate the ratio epithelial signal over leukocyte signal

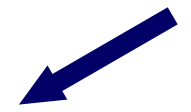

5 a Samples with detectable epithelial-derived signals
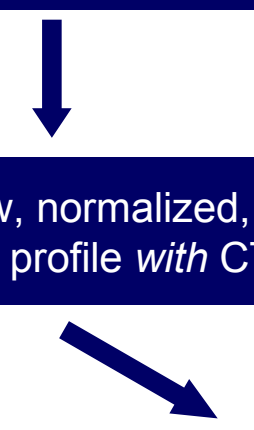

7 Analyze only transcripts more highly expressed in samples with detectable epithelial-derived signals, i.e. from CTCs 5b Samples from healthy blood donors

6b Cut-off, normalized, expression profile without CTCs

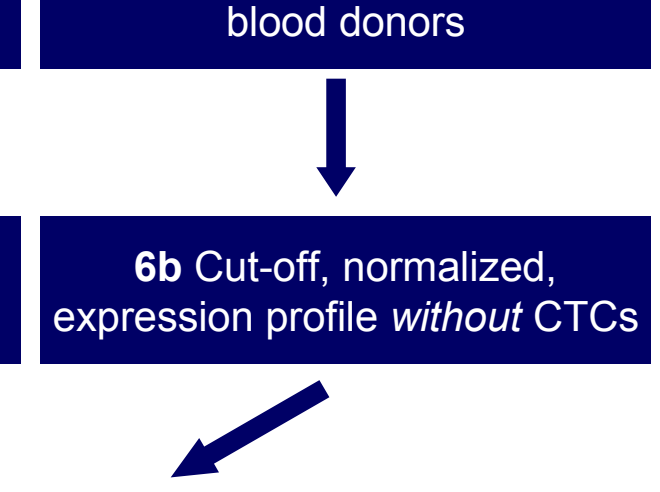
es

\section{6a Raw, normalized, \\ expression profile with CTCs}




\section{one-stop shop}

\section{Baseline}

\section{Follow-up}

\section{CTC
enumeration \\ CTC
enumeration}

CTC isolation
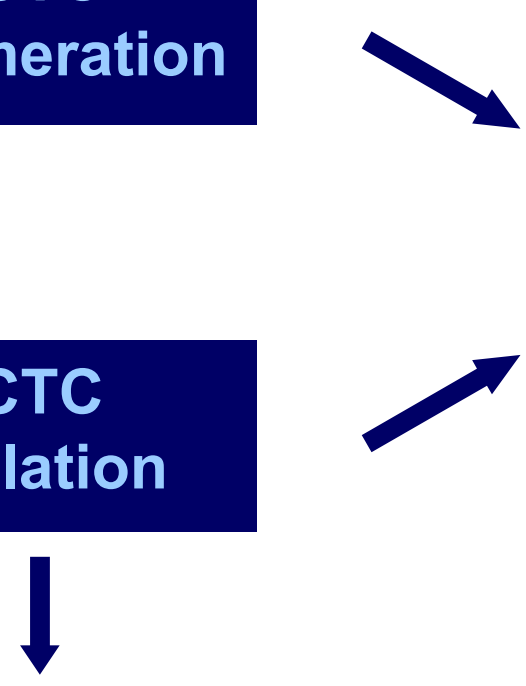

- gDNA mutation or methylation?

- mRNA/miRNA expression?

- receptor status?

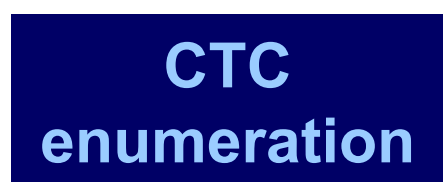

Choose therapy
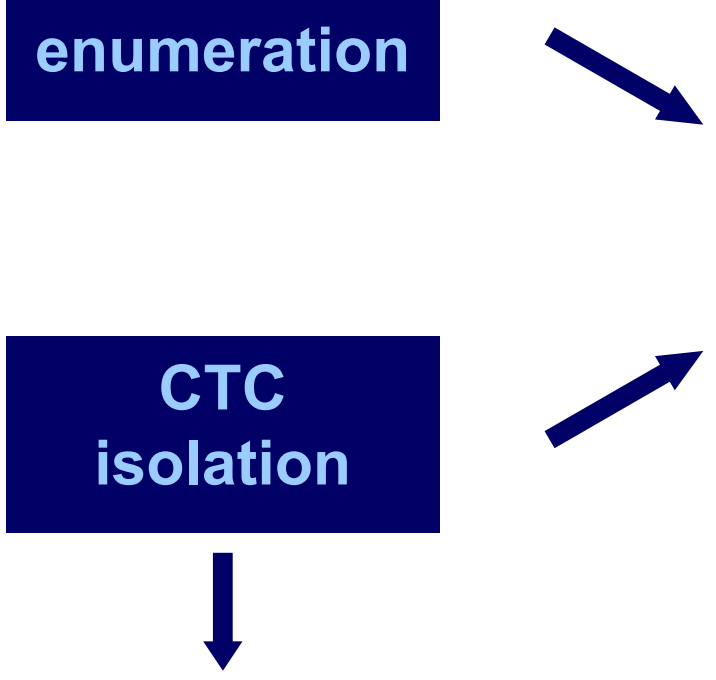

- acquired or loss of gDNA mutation?

- altered mRNA/miRNA expression?

- switch in receptor status? 